\title{
Accurate forced-choice recognition without awareness of memory retrieval
}

\author{
Joel L. Voss, ${ }^{1,2,4}$ Carol L. Baym, ${ }^{1,2,3}$ and Ken A. Paller ${ }^{1,2}$ \\ ${ }^{1}$ Interdepartmental Neuroscience Program, Northwestern University, Evanston, Illinois 60208-2710, USA; ${ }^{2}$ Department of \\ Psychology, Northwestern University, Evanston, Illinois 60208-2710, USA
}

\begin{abstract}
Recognition confidence and the explicit awareness of memory retrieval commonly accompany accurate responding in recognition tests. Memory performance in recognition tests is widely assumed to measure explicit memory, but the generality of this assumption is questionable. Indeed, whether recognition in nonhumans is always supported by explicit memory is highly controversial. Here we identified circumstances wherein highly accurate recognition was unaccompanied by hallmark features of explicit memory. When memory for kaleidoscopes was tested using a two-alternative forced-choice recognition test with similar foils, recognition was enhanced by an attentional manipulation at encoding known to degrade explicit memory. Moreover, explicit recognition was most accurate when the awareness of retrieval was absent. These dissociations between accuracy and phenomenological features of explicit memory are consistent with the notion that correct responding resulted from experience-dependent enhancements of perceptual fluency with specific stimuli-the putative mechanism for perceptual priming effects in implicit memory tests. This mechanism may contribute to recognition performance in a variety of frequently-employed testing circumstances. Our results thus argue for a novel view of recognition, in that analyses of its neurocognitive foundations must take into account the potential for both (1) recognition mechanisms allied with implicit memory and (2) recognition mechanisms allied with explicit memory.
\end{abstract}

An important principle in memory research is that there are characteristic behavioral, cognitive, subjective, and neural features that set explicit memory apart from other expressions of memory (Gabrieli 1998; Squire 2004; Paller et al. 2008). Explicit memory tests are those wherein direct reference is made to prior learning events, such as when subjects deliberately discriminate repeat from novel items in a recognition test. Memory measured by such tests is referred to as explicit memory. Hallmark features of explicit memory include the awareness of memory retrieval and confidence in memory judgments. Explicit memory can be contrasted with another form of memory, implicit memory, which does not require the realization that memory has influenced behavior. Specialized tests in which no reference is made to prior learning episodes are commonly used to measure implicit memory. Tests of perceptual priming, for example, are used to measure implicit memory for physical stimulus features, and perceptual priming with visual stimuli is thought to be supported by repetition-induced enhancements in neural processing fluency within visual cortex (Wiggs and Martin 1998; Schacter et al. 2007).

A central emphasis of contemporary memory research has been to determine how explicit memory and implicit memory are related. Many researchers have proposed that recognition may derive partly from repetition-based perceptual fluency, the same mechanism that mediates perceptual priming (e.g., Jacoby and Dallas 1981; Johnston et al. 1991; Verfaellie and Cermak 1999; Yonelinas 2002). The primary difference may be that recognition requires fluency plus attribution of prior occurrence, whereas no such attribution of prior occurrence is required for priming. One way fluency has been studied is with stimuli slowly emerging from noise (gradual unmasking), wherein the speed or fluency of item identification appears to be related to the tendency to report that the item had been seen previously (Verfaellie

${ }^{3}$ Present address: Department of Psychology, University of Illinois, Urbana-Champaign, Champaign, IL 61820-6232, USA.

${ }^{4}$ Corresponding author.

E-mail joel-voss@northwestern.edu; fax (847) 491-7859.

Article is online at http://www.learnmem.org/cgi/doi/10.1101//m.971208. and Cermak 1999; Conroy et al. 2005). Other methods have also been used to show that fluency can provoke "old" responses in recognition tests (e.g., Jacoby and Whitehouse 1989; Whittlesea and Williams 2000; Kleider and Goldinger 2004). Importantly, a bias to call fluently processed stimuli "old" can readily occur for stimuli not previously viewed (e.g., Verfaellie and Cermak 1999). Given that perceptual fluency can derive from sources other than repetition, it may not always promote accurate recognition. But do subjects use it anyway, under some circumstances? Whether subjects adopt a fluency-based heuristic for responding may vary depending on factors such as the way they approach the task, the strength of context-rich episodic recall, learned habits, and whether fluency is likely to distinguish old items from new ones (Whittlesea and Price 2001; Keane et al. 2006). For example, a relative amelioration of the recognition deficit in amnesic patients was found in a special recognition test in which fluency was higher for old compared to new words due to the use of systematically different letters in old versus new words (Keane et al. 2006). Clearly, then, tests can be constructed such that subjects do use fluency as an accurate cue to recognition. On the other hand, findings of normal implicit memory in amnesic patients with little or no capacity for explicit memory (e.g., Hamann and Squire 1997; Stark and Squire 2000) suggest that explicit memory relies on neural mechanisms distinct from perceptual fluency. Furthermore, repetition-based fluency in a standard recognition test appeared to be insufficient to drive recognition much above chance levels (Conroy et al. 2005). However, a general conclusion about the role of perceptual fluency may not be warranted, because the role may vary depending on the way memory is tested. All in all, we currently lack a full understanding of the extent to which fluency can drive accurate recognition for some items, and under what testing circumstances.

Neuroanatomical substrates of explicit memory are commonly studied using recognition tests. In memory research with nonhuman animals, vigorous controversy surrounds whether recognition as assessed in these tests relies on explicit memory mechanisms analogous to those that support recognition in humans (Clayton et al. 2003; Hampton and Schwartz 2004). Be- 


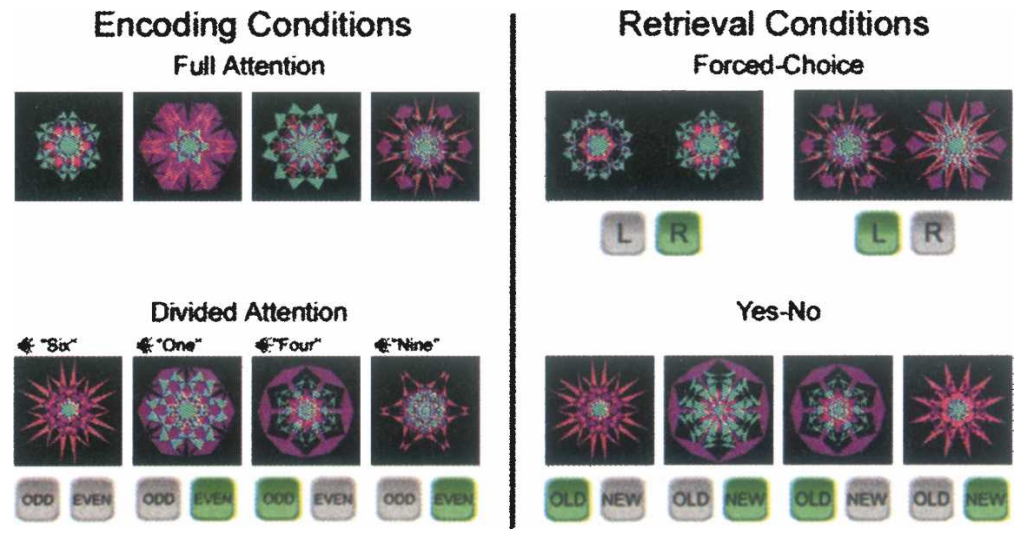

Figure 1. Schematic representation of encoding and retrieval conditions. Icons show the two possible button-press responses for each trial, with correct responses highlighted in green. Subjects encoded kaleidoscopes with either full attention or with divided attention. With divided attention, participants performed an auditory one-back task with odd/even judgments to spoken numeric digits. The recognition-testing format was either two-alternative forced-choice or yes-no. A within-subjects factorial design was used to assign the study and test conditions to the four study-test blocks, in counterbalanced order.

cause the animals cannot verbally communicate subjective features of memory, some of the hallmark features of human explicit memory, such as the awareness of remembering and metamemory expressions of recognition confidence, are difficult to verify.

We sought to determine whether there are circumstances in which human recognition is supported by memory processes that are aligned with implicit memory and that do not entail these hallmark features of explicit memory. We utilized kaleidoscope stimuli such that verbal encoding and retrieval strategies that typically foster response confidence and retrieval awareness were ineffective. To potentially implicate explicit memory, attentional resources during encoding were manipulated, based on prior findings that reduced attention leads to reduced explicit memory, including both recollection, wherein pertinent details from initial stimulus presentation are retrieved, and familiarity, wherein a feeling of recognition occurs without specific episodic retrieval (Mulligan 1998; Rajaram et al. 2001; Yonelinas 2001; Curran 2004). Although it might be argued that we used atypical recognition testing circumstances, the properties of our memory tests were similar to those of recognition tests often given to nonhuman animals; stimuli were nearly meaningless and not likely to be remembered using verbal strategies. We hypothesized that the properties of recognition memory in these circumstances would be suitable for determining whether forced-choice recognition can be accomplished via mechanisms distinct from those commonly thought to support explicit memory. This approach is thus aimed at understanding the full range of neurocognitive processes that can support recognition memory. yes-no tests). Bars indicate mean and error bars indicate SEM.

\section{Results}

In Experiment 1, recognition memory for kaleidoscope stimuli was probed using two test formats (Fig. 1). Recognition accuracy was robustly influenced by whether encoding occurred under full attention or during the concurrent performance of a working-memory task that required odd/even judgments on spoken digits. This effect of attention at encoding on memory performance (Fig. 2A) varied systematically with test format, as indicated by a crossover interaction $\left(F_{(1,23)}=36.1, P<0.001\right)$. The drastic reduction in yes-no performance to nearchance levels for divided compared to full attention $\left(t_{(23)}=3.9, P<0.001\right)$ clearly implicates explicit-memory processes.

Strikingly, memory was significantly improved for encoding with divided compared to full attention when recognition was tested using the forcedchoice format $\left(t_{(23)}=4.0, P<0.001\right)$. This improvement is the opposite of what would be expected if explicit memory were driving performance. This pattern of results is therefore suggestive of a mechanism operative for forced-choice performance that is distinct from the explicit-memory processes operative for yes-no recognition.

To determine the metamemory concomitants of this unusual increase in memory accuracy with poorer encoding, we

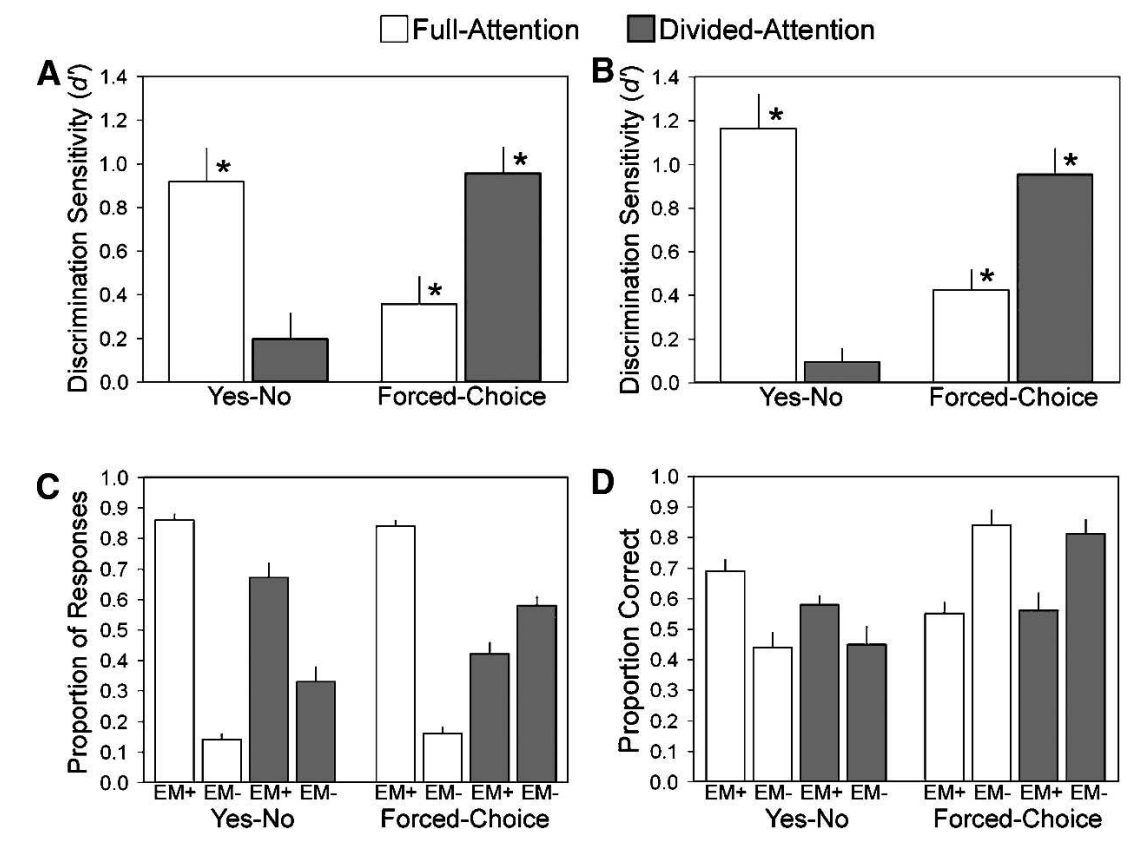

Figure 2. Memory accuracy varied with study-phase attentional resources, test format, and metamemory judgments. (A) Forced-choice and yes-no performance were differentially affected by divided attention during encoding in Experiment 1. Asterisks indicate that recognition sensitivity $\left(d^{\prime}\right)$ was significantly above chance (higher than zero, $P<0.05$ ). (B) A similar pattern of recognition results was obtained in Experiment 2. (C) Trial breakdown for Experiment 2 is shown according to whether there was some degree of explicit-memory confidence $(\mathrm{EM}+)$ versus no explicit-memory confidence $(E M-)$, computed separately for each encoding condition and test condition. (D) Recognition accuracy for Experiment 2 is shown, computed separately for each metamemory condition (EM+ or EM -, as in C), encoding condition, and test condition (percent correct for forced-choice and for old items in 
Table 1. Mean recognition scores as proportion correct for each condition

\begin{tabular}{|c|c|c|c|}
\hline & \multirow{2}{*}{$\begin{array}{l}\text { Forced-choice } \\
\text { recognition }\end{array}$} & \multicolumn{2}{|c|}{ Yes-no recognition } \\
\hline & & Old & New \\
\hline \multicolumn{4}{|l|}{ Experiment 1} \\
\hline Full-attention & $0.59(0.03)$ & $0.66(0.03)$ & $0.65(0.0$ \\
\hline Divided-attention & $0.72(0.03)$ & $0.51(0.02)$ & $0.56(0.03)$ \\
\hline \multicolumn{4}{|l|}{ Experiment 2} \\
\hline Full-attention & $0.61(0.03)$ & $0.66(0.04)$ & $0.74(0.0$ \\
\hline Divided-attention & $0.73(0.03)$ & $0.50(0.03)$ & $0.53(0.03)$ \\
\hline \multicolumn{4}{|l|}{ Experiment 3} \\
\hline Full-attention & $0.72(0.03)$ & $0.59(0.02)$ & $0.72(0.02)$ \\
\hline Divided-attention & $0.69(0.03)$ & $0.55(0.02)$ & $0.63(0.03)$ \\
\hline \multicolumn{4}{|l|}{ Experiment 4} \\
\hline Full-attention & $0.66(0.03)$ & & \\
\hline Divided-attention & $0.61(0.03)$ & & \\
\hline
\end{tabular}

SEM in parentheses.

administered the same recognition tests to another group of subjects who were required to introspectively assess the awareness of recognition on each test trial (Experiment 2). Ratings on a three-point confidence scale were collapsed into two categories that indicated the presence of subjective explicit memory $(\mathrm{EM}+)$ or the absence of subjective explicit memory (EM-). Figure $2 \mathrm{~B}$ shows that the pattern of recognition performance from Experiment 1 was replicated, with the same crossover interaction between attention at encoding and test format $\left(F_{(1,23)}=68.7\right.$, $P<0.001)$. Divided compared to full attention at encoding yielded a reduction in yes-no performance $\left(t_{(23)}=6.6, P<0.001\right)$ and an increase in forced-choice performance $\left(t_{(23)}=4.0\right.$, $P<0.001)$. This novel pattern of results was thus highly reliable (the same effects of attention at encoding appeared in these two experiments as well as in a pilot study with another group of subjects, when attention was manipulated between groups to yield the same crossover interaction $\left.\left[F_{(1,18)}=39.2, P<0.001\right]\right)$.

Metamemory findings provided an additional empirical dimension in Experiment 2 (Fig. 2C). Consonant with prior findings (Yonelinas 2001; Curran 2004), a downward shift in recognition confidence was apparent following divided compared to full attention in both tests $\left(F_{(1,23)}=75.8, P<0.001\right)$. In other words, EM+ responses became less prevalent with divided attention. This shift was more pronounced for the forced-choice compared to the yes-no format $\left(F_{(1,23)}=21.6, P<0.001\right)$.

It may seem counterintuitive that attentional load at study simultaneously decreased forced-choice recognition confidence and increased accuracy. However, this pattern makes sense when considering forced-choice accuracy as a function of confidence level; accuracy was maximal when subjects thought they were guessing (Fig. 2D). Forced-choice accuracy was significantly higher for $\mathrm{EM}$ - trials than for $\mathrm{EM}+$ trials $\left(t_{(23)}=3.2, P=0.004\right)$. Thus, attentional load at study led to improved forced-choice recognition because it amplified the number of guess trials, and in those trials forced-choice accuracy was remarkably high.

In the yes-no test, the attentional load reduced both accuracy and confidence. In keeping with the intuitive notion that accuracy and confidence should go together, yes-no accuracy was highest for EM+ responses (Fig. 2D). The relationship between yes-no accuracy and confidence was assessed by subjecting proportion-correct scores to an ANOVA with three factors: repetition (old/new), encoding condition (divided/full attention), and confidence $(\mathrm{EM}+/ \mathrm{EM}-)$. A main effect of confidence $\left(F_{(1,23)}=61.9\right.$, $P<0.001)$ indicated that accuracy decreased along with confidence for both old and new items and for both encoding conditions (no other main effects or interactions reached statistical significance). Within each confidence level, recognition accuracy did not differ as a function of attentional load at encoding (forced-choice $F_{(1,23)}=0.36$, not significant [ns]; yes-no $\left.F_{(1,23)}=0.18, \mathrm{~ns}\right)$, showing that metamemory judgments indexed memory abilities to the same degree across all conditions.

Unlike yes-no recognition, accurate forced-choice recognition was supported primarily by EM - responses, when explicit memory-from the subject's point of view-was completely absent. The benefit of divided-attention encoding on forced-choice performance can therefore be attributed to the deleterious effect of this manipulation on explicit memory.

Recognition sensitivity $\left(d^{\prime}\right)$ scores were used in these analyses so that performance in yes-no and forced-choice tests could be directly compared (Macmillan and Creelman 2005). The same patterns of effects described herein were also clear in hit rates (Table 1). Differences in sensitivity between yes-no and forcedchoice tests were not due to differences in the bias to respond "old" $(c)$, because $c=0$ on forced-choice tests and $c$ did not differ from zero on yes-no tests (Experiment 1, full attention mean $=-0.02$, divided attention mean $=0.07$; Experiment 2, full attention mean $=0.11$, divided attention mean $=0.05$; $t_{(23)} \leq 1.3$, ns in all cases). Even though response bias was similar, there are several disparities between yes-no and forced-choice format tests that complicate direct comparisons between overall performance levels (Bayley et al. 2008), including differences in study/test delays, order of targets and foils, and the setting of response criteria for recognition decisions. Our interpretations, however, turn on the findings from the forced-choice test, not on the contrast between the two types of test.

Notably, recognition responses in Experiments 1 and 2 were made $\sim 2 \mathrm{sec}$ after stimulus onset, so there was moderate but not severe time pressure. Also, the perceptual similarity of targets and foils was extremely high. Both of these design features were intended to limit the influence of explicit memory on recognition. In Experiments 3 and 4 we altered these parameters in order to probe boundary conditions for our novel findings.

Experiment 3 was identical to Experiment 1 except that kaleidoscopes remained on the screen until recognition responses were made, and subjects were encouraged to respond at their leisure and to carefully evaluate the two choices before responding. We hypothesized that contributions from explicit memory would be enhanced to the extent that subjects attempted to engage in effortful memory search and evaluation. Response time averaged $7.5 \mathrm{sec}$ and did not differ significantly as a function of test format, encoding condition, repetition (old vs. new for yesno tests only), or accuracy ( $P>0.19$ for all ANOVA main effects and interactions). Figure $3 \mathrm{~A}$ shows that performance in both yesno and forced-choice tests declined with divided attention

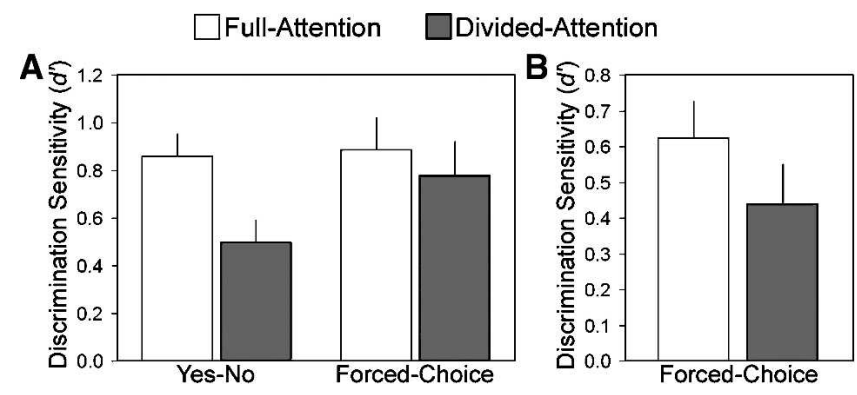

Figure 3. No evidence for contributions from the novel recognition process to performance in Experiments 3 and 4. (A) In Experiment 3, time pressure was not imposed. Recognition accuracy was reduced by divided attention at encoding for both forced-choice and yes-no tests. (B) In Experiment 4, forced-choice performance with targets paired with noncorresponding foils was marginally reduced by divided attention at encoding. Bars indicate mean and error bars indicate SEM. 
$\left(F_{(1,23)}=5.0, P=0.04\right.$, nonsignificant test-type interaction, $\left.F_{(1,23)}=1.35\right)$.

Experiment 4 was composed of two additional study-test blocks with forced-choice format tests following Experiment 3. In these blocks, encoding and test parameters were identical to those in Experiment 1 (including time pressure), except that the 10 targets and 10 foils were paired such that each target appeared with a foil other than the corresponding foil. Previous experiments with visual scenes have shown that mismatched target/foil pairings can lead to reduced accuracy with greater confidence compared to more perceptually similar target/foil pairs (Tulving 1981; Dobbins et al. 1998). We thus hypothesized that randomly assigning targets and foils would decrease recognition accuracy overall. Furthermore, we reasoned that this manipulation would enhance the utility of explicitly retrieving specific details from the encoding phase. Recognition accuracy was numerically worse for divided compared to full attention (Fig. 3B), although this trend did not reach statistical significance $\left(t_{(23)}=1.2\right.$, ns). Thus, there was no evidence that the novel recognition mechanism contributed to performance in either Experiment 3 or Experiment 4.

\section{Discussion}

Recognition memory was assessed using procedures designed specifically to facilitate responding in the absence of explicit memory. Recognition tests were constructed using pairs of kaleidoscope stimuli exhibiting a high degree of visual similarity between the two members of each pair. It was thus difficult to discriminate targets from foils based on color or other perceptual features, or based on conceptual content. Moderate pressure to make responses quickly further discouraged reliance on explicit memory. Each experiment included a manipulation to reduce attention at encoding. In contradistinction to the robust explicitmemory disruption generally found with this manipulation, we found that forced-choice recognition accuracy was enhanced by reduced attention at encoding. The fact that yes-no recognition was less accurate following divided-attention encoding compared to full-attention encoding shows that our manipulation of attention at encoding can produce the typical effect on later memory, and also substantiates the notion that the yes-no test measured standard explicit-memory processing.

Importantly, responses in the forced-choice test were highly accurate when explicit memory was introspectively absent (Experiment 2). Given this dissociation between memory accuracy and metamemory, and the unusual relationship between forcedchoice recognition and attention at encoding, we infer that novel recognition-memory processes supported accurate forced-choice performance.

Upon debriefing, subjects often reported finding the kaleidoscope memory tests very difficult. Nevertheless, unusually high recognition accuracy-over $80 \%$ correct, on average-was found when people thought they were unable to retrieve information sufficient to make anything more than a guess (EMresponses in Experiment 2). This dissociation between accuracy and first-person experience is reminiscent of several phenomena in neuropsychology. For example, patients with blindsight are sometimes able to make accurate two-alternative visual discrimination judgments when they claim to be unable to see the stimuli in question (Weiskrantz et al. 1995). Similar phenomena have been demonstrated in healthy subjects (Lau and Passingham 2006) and in monkeys (Cowey and Stoerig 1995). Here, we found that people had remarkably good recognition abilities when conscious memory retrieval apparently failed.

We speculate that the novel recognition mechanism was composed of a memory influence based on perceptual fluency of the variety that normally supports performance on tests of per- ceptual implicit memory. Repeat items may be selected in forcedchoice tests because the fluency with which they are perceived is systematically greater than that of the corresponding novel items. This cue to recognition is effectively eliminated in yes-no tests because targets are not presented together with corresponding foils. Although the forced-choice test with two nearly identical stimuli on each trial appears highly difficult to subjects, this test affords a special opportunity for subtle differences in perceptual fluency to drive accurate recognition, particularly in the absence of strong explicit memory. Further comparisons across a variety of testing circumstances, including implicit memory testing, will be helpful for understanding how perceptual fluency influences memory performance.

We suggest that explicit memory in our experiments was reduced by divided attention at encoding whereas implicit memory may have been unaffected or only mildly reduced. In prior studies, divided attention during encoding has been found to disproportionately influence explicit memory compared to implicit memory. Indeed, priming is often unaffected by divided attention despite marked reductions in explicit memory (e.g., Mulligan 1998). Other studies have indicated that divided attention reduces priming to a far lesser extent than explicit memory (e.g., Rajaram et al. 2001). These relationships concern implicit memory for perceptual stimulus attributes (perceptual priming), whereas conceptual priming is reduced by divided attention to a similar extent as explicit memory (Mulligan 1998; Light et al. 2000). Moreover, behavioral estimates of explicit familiarity are reduced by divided attention, but to a lesser extent than estimates of explicit recollection (Yonelinas 2001). Although it may be tempting to assume that implicit memory was strengthened by divided-attention encoding in our experiments, such an assumption is not necessary to explain enhanced performance for this condition. Rather, we postulate that subjects tended to engage different strategies in divided- and full-attention conditions and so relied on different processes to drive recognition responding. The potential for conscious access to relevant information from study episodes was presumably much less following divided-attention encoding, and guessing was more common. Is it possible that memory processes that typically support priming in implicit memory tests were operative when subjects felt they were guessing? The EM - trials identified in Experiment 2 may be diagnostic of a novel recognition mechanism wherein high recognition accuracy is achieved with negligible confidence. The finding that accuracy for EM - trials was very similar for the two attention conditions is consistent with the notion that recognition responding was driven by perceptual implicit memory to the same extent regardless of level of attention at encoding. Given that the prevalence of EM - trials increased with divided attention, under these circumstances subjects may have preferentially relied on a fluency-based strategy that does not entail awareness of remembering.

A related way to explain our novel findings is to speculate that accurate recognition after divided-attention encoding reflects familiarity memory. Indeed, forced-choice recognition tests with highly similar foils are known to encourage strategies of assessing the relative strength of familiarity signals engendered by each stimulus (Aggleton and Shaw 1996; Khoe et al. 2000; O'Reilly and Rudy 2000; Holdstock et al. 2002; Yonelinas 2002; Bastin and Van der Linden 2003; Westerberg et al. 2006). Nonetheless, disentangling neural signals of familiarity memory versus implicit memory is difficult because during recognition testing both can occur concurrently (Paller et al. 2007). Given that familiarity is characterized as relatively rapid and automatic compared to recollection (Yonelinas 2002), it is conceivable that familiarity was preferentially involved in our experiments because of the pressure to respond quickly. It is thus important to be clear 
about how we conceptualize familiarity memory. Familiaritybased recognition is taken as an instance of explicit memory because familiarity responses entail the awareness of memory retrieval (e.g., "this stimulus feels familiar in that it seems like it must have been shown to me earlier"). If, on the other hand, familiarity-based recognition responses are conceived of as independent of conscious feelings of familiarity, then familiarity could transpire either in conjunction with or in the absence of the subjective experience of recognition. However, we would advocate for reserving the term familiarity for recognition experiences closely tied to the conscious experience of familiarity, consistent with the most common usage of this term. Given that the novel recognition mechanism observed here was coupled more closely with negligible recognition confidence than with any higher level of recognition confidence, we infer that it is not allied with familiarity memory. Rather, recognition was likely to have resulted from perceptual fluency without any phenomenological awareness of recognition, perhaps the very same fluency process that can lead to priming in perceptual implicit memory tests.

Despite the restricted testing parameters examined in the present experiments, our results indicate that recognition can be supported by memory processing dissimilar to explicit memory when (1) elaborative conceptual encoding is minimal; (2) manipulations that reduce explicit memory are employed; (3) memory for spatiotemporal features of the learning episode are unhelpful for choosing the correct answer; and (4) forced-choice responses are made quickly or automatically. Similar inverse relationships between recognition confidence and recognition accuracy have been identified in memory for visual scenes (Tulving 1981; Dobbins et al. 1998), although these studies did not find evidence that accurate responding occurred without awareness of memory retrieval. Other results have also suggested that perceptual fluency is most likely to influence recognition when explicit memory strength is minimal (Johnston et al. 1991; Tunney and Fernie 2007).

Some patients with memory disorders have exhibited preserved recognition in forced-choice tests using targets and foils with high perceptual similarity (Holdstock et al. 2002; Westerberg et al. 2006). Likewise, recognition performance in monkeys can be unimpaired after hippocampal lesions (Baxter and Murray 2001). In both cases, recognition was presumably guided more by perceptual information than by conceptual information. Other findings from monkeys and humans have been taken as support for the hypothesis that recollection relies on the hippocampus whereas familiarity relies on the adjacent medial temporal cortex (Aggleton and Brown 2006). However, some evidence speaks against this dichotomy by indicating that the human hippocampus is critical for both recollection and familiarity (Wixted and Squire 2004; Wais et al. 2006; Squire et al. 2007). The validity of a simple dichotomy is further weakened by the present findings, which indicate that forced-choice performance in some circumstances may not be driven solely by explicit-memory processing.

Nonhuman animals commonly provide model systems for probing the neuroanatomical substrates of what is considered to be explicit (or "episodic-like") memory (Aggleton and Pearce 2002; Squire et al. 2004). The validity of this approach depends on close connections between corresponding memory functions across species. Unlike humans, nonhuman animals must undergo extensive training on the tasks used to probe memory, thus allowing them to discover optimal performance strategies to maximize rewards. The present findings are in keeping with the proposition that some neuroscience experiments attempting to model explicit memory in animals might not actually be scrutinizing explicit memory (Clayton et al. 2003; Hampton and Schwartz 2004), in that our results indicate that forced-choice recognition tests for stimuli of low conceptual content might not scrutinize explicit memory in humans. Future studies of recognition in both humans and nonhuman animals will be strengthened to the extent that they account for the multiple memory processes that can drive accurate forced-choice recognition, including those in the category of explicit memory as well as those in the category of implicit memory.

\section{Materials and Methods}

\section{Experiment 1}

Visual stimuli included 80 kaleidoscope images divided into 40 perceptually similar target/foil pairs (Fig. 1). Each pair was created by initially overlaying three hexagons, each of a different color (four different sets were created, each with unique colors). Each hexagon was then altered by three rounds of side bisection and deflection at a random direction (up to $10^{\circ}$ different for each member of a target/foil pair). For each pair, one image was randomly selected as target for each subject. An additional 16 kaleidoscopes were used as fillers. Stimuli subtended $4^{\circ}-6^{\circ}$ of visual angle and were presented on a black background.

Subjects $(N=24$, ages $18-22 \mathrm{yr}, 13$ male) performed four study-test blocks wherein they encoded kaleidoscopes and then discriminated repeat images (targets) from novel images (foils). At study, 14 kaleidoscopes were presented for $2000 \mathrm{msec}$, one every 3500 msec. In each block, all kaleidoscopes had the same three colors. Primacy and recency effects were reduced by not testing the first two and last two study-phase kaleidoscopes (fillers). At test, 10 targets were mixed with 10 corresponding foils.

A within-subject factorial design was used with two studyphase conditions and two test-phase conditions (order of conditions counterbalanced across subjects). Subjects were neither informed of the test order nor the total number of blocks, such that the format of the upcoming test could not be determined during encoding.

Encoding was performed under divided attention (two blocks) or full attention (two blocks). Subjects attempted to memorize each kaleidoscope either with or without a concomitant one-back task, respectively. The task involved a random series of prerecorded spoken numeric digits, one presented when each kaleidoscope appeared. On each trial (except the first), subjects pressed a button to indicate whether the previous digit was odd or even.

Recognition was tested using a yes-no format (two blocks) or a forced-choice format (two blocks). In each yes-no trial, a kaleidoscope appeared for $2000 \mathrm{msec}$, with button choices to indicate "old" (target) or "new" (foil). In each forced-choice trial, a target/ foil pair appeared together for $2000 \mathrm{msec}$, with button choices to indicate the side of the target. The target was equally likely to appear on the left or right side. Subjects were required to respond immediately after images disappeared from the screen, thus imposing an effective response deadline of about 2 sec from stimulus onset. Response latency was monitored by the experimenter, and subjects were reminded to adhere to the deadline when necessary. After the response, there was a 1000-msec delay before the next trial. The total retention delay was $\sim 2 \mathrm{~min}$.

\section{Experiment 2}

Visual stimuli and procedures were identical to those in Experiment 1 with the following exceptions. Subjects $(N=24$, ages 18 $25 \mathrm{yr}, 10$ male) were cued to report recognition confidence following every trial. (Subjects in each experiment had not participated in any of the previous experiments.) The cue to make a metamemory judgment appeared directly following the recognition response, and was composed of a display of a three-point confidence scale, which was intended to segregate memory with awareness from memory without awareness. The high-confidence button indicated confidence due to retrieval of specific details from the study phase supporting the recognition decision. The low-confidence button indicated recognition supported only by "a weak feeling of familiarity" with no details from the study phase retrieved. The guess button indicated "absolutely no feeling of memory" such that the stimulus "in no way felt 'old'." Subjects 
were told to press this button when they were "just randomly guessing because the experimenter is forcing you to make a response." This scale is not taken to provide estimates of recollection and familiarity, because of the emphasis on both response confidence and subjective awareness. The scale is nonetheless advantageous in that it permits a metamemory-based categorization of trials according to the degree to which explicit memory contributed to performance. Accordingly, we collapsed highconfidence and low-confidence responses into a single category for which subjective explicit memory was present $(\mathrm{EM}+)$, whereas guess responses indicated that subjective explicit memory was absent (EM-).

\section{Experiments 3 and 4}

Subjects ( $N=24$, ages $18-27$ yr, 13 male) performed Experiment 3 followed immediately by Experiment 4 . Different kaleidoscopes were used in the two experiments. Experiment 3 was identical to Experiment 1, except that kaleidoscopes remained on the screen until recognition responses were made. Subjects were encouraged to respond at their leisure and to engage in effortful retrieval before responding. Experiment 4 included two study-test blocks with forced-choice format tests. Encoding was with either divided or full attention, in counterbalanced order. Encoding and test parameters were identical to Experiment 1 (including time pressure), except that the 10 targets and 10 foils were paired such that each foil appeared with a target other than the corresponding target.

\section{Acknowledgments}

We thank Antonio Gisbert and Paul Reber for their assistance in generating stimuli, and Courtney Clark and Olivia Marczuk for assistance with data collection. This work was supported by grants from the United States National Institutes of Health (R01NS34639, P30-AG13854, and T32-AG20506) and the National Science Foundation (0518800).

\section{References}

Aggleton, J.P. and Brown, M.W. 2006. Interleaving brain systems for episodic and recognition memory. Trends Cogn. Sci. 10: 455-463.

Aggleton, J.P. and Pearce, J.M. 2002. Neural systems underlying episodic memory: Insights from animal research. In Episodic memory: New directions in research (eds. M.A. Conway et al.). 204-231. Oxford, New York.

Aggleton, J.P. and Shaw, C. 1996. Amnesia and recognition memory: A re-analysis of psychometric data. Neuropsychologia 34: 51-62.

Bastin, C. and Van der Linden, M. 2003. The contribution of recollection and familiarity to recognition memory: A study of the effects of test format and aging. Neuropsychology 17: 14-24.

Baxter, M.G. and Murray, E.A. 2001. Opposite relationship of hippocampal and rhinal cortex damage to delayed nonmatching-to-sample deficits in monkeys. Hippocampus 11: 61-71.

Bayley, P.J., Wixted, J.T., Hopkins, R.O., and Squire, L.R. 2008. Yes/no recognition, forced-choice recognition, and the human hippocampus. J. Cogn. Neurosci. 20: 505-512.

Clayton, N.S., Bussey, T.J., and Dickinson, A. 2003. Can animals recall the past and plan for the future? Nat. Rev. Neurosci. 4: 685-691.

Conroy, M.A., Hopkins, R.O., and Squire, L.R. 2005. On the contribution of perceptual fluency and priming to recognition memory. Cogn. Affect. Behav. Neurosci. 5: 14-20.

Cowey, A. and Stoerig, P. 1995. Blindsight in monkeys. Nature 373: $247-249$.

Curran, T. 2004. Effects of attention and confidence on the hypothesized ERP correlates of recollection and familiarity. Neuropsychologia 42: 1088-1106.

Dobbins, I.G., Kroll, N.E., and Liu, Q. 1998. Confidence-accuracy inversions in scene recognition: A remember-know analysis. J. Exp. Psychol. Learn. Mem. Cogn. 24: 1306-1315.

Gabrieli, J.D. 1998. Cognitive neuroscience of human memory. Annu. Rev. Psychol. 49: 87-115.

Hamann, S.B. and Squire, L.R. 1997. Intact perceptual memory in the absence of conscious memory. Behav. Neurosci. 111: 850-854.

Hampton, R.R. and Schwartz, B.L. 2004. Episodic memory in nonhumans: What, and where, is when? Curr. Opin. Neurobiol. 14: $192-197$.

Holdstock, J.S., Mayes, A.R., Roberts, N., Cezayirli, E., Isaac, C.L., O'Reilly, R.C., and Norman, K.A. 2002. Under what conditions is recognition spared relative to recall after selective hippocampal damage in humans? Hippocampus 12: 341-351.
Jacoby, L.L. and Dallas, M. 1981. On the relationship between autobiographical memory and perceptual learning. J. Exp. Psychol. Learn. Mem. Cogn. 110: 306-340.

Jacoby, L.L. and Whitehouse, K. 1989. An illusion of memory: False recognition influenced by unconscious perception. J. Exp. Psychol. Gen. 118: 126-135.

Johnston, W.A., Hawley, K.J., and Elliott, J.M. 1991. Contribution of perceptual fluency to recognition judgments. J. Exp. Psychol. Learn. Mem. Cogn. 17: 210-223.

Keane, M.M., Orlando, F., and Verfaellie, M. 2006. Increasing the salience of fluency cues reduces the recognition memory impairment in amnesia. Neuropsychologia 44: 834-839.

Khoe, W., Kroll, N.E., Yonelinas, A.P., Dobbins, I.G., and Knight, R.T. 2000. The contribution of recollection and familiarity to yes-no and forced-choice recognition tests in healthy subjects and amnesics. Neuropsychologia 38: 1333-1341.

Kleider, H.M. and Goldinger, S.D. 2004. Illusions of face memory: Clarity breeds familiarity. J. Mem. Lang. 50: 196-211.

Lau, H.C. and Passingham, R.E. 2006. Relative blindsight in normal observers and the neural correlate of visual consciousness. Proc. Natl. Acad. Sci. 103: 18763-18768.

Light, L.L., Prull, M.W., and Kennison, R.F. 2000. Divided attention, aging, and priming in exemplar generation and category verification. Mem. Cognit. 28: 856-872.

Macmillan, N.A. and Creelman, C.D. 2005. Detection theory: A user's guide, $2 \mathrm{~d}$ ed. Lawrence Erlbaum Associates, Mahwah, NJ.

Mulligan, N.W. 1998. The role of attention during encoding in implicit and explicit memory. J. Exp. Psychol. Learn. Mem. Cogn. 24: 27-47.

O'Reilly, R.C. and Rudy, J.W. 2000. Computational principles of learning in the neocortex and hippocampus. Hippocampus 10: $389-397$.

Paller, K.A., Voss, J.L., and Boehm, S.G. 2007. Validating neural correlates of familiarity. Trends Cogn. Sci. 11: 243-250.

Paller, K.A., Voss, J.L., and Westerberg, C.E. 2008. Memory and the awareness of remembering. In Neuroimaging of human memory (eds. F. Rösler et al.). Oxford University Press, Oxford, UK. (in press).

Rajaram, S., Srinivas, K., and Travers, S. 2001. The effects of attention on perceptual implicit memory. Mem. Cognit. 29: 920-930.

Schacter, D.L., Wig, G.S., and Stevens, W.D. 2007. Reductions in cortical activity during priming. Curr. Opin. Neurobiol. 17: 171-176.

Squire, L.R. 2004. Memory systems of the brain: A brief history and current perspective. Neurobiol. Learn. Mem. 82: 171-177.

Squire, L.R., Stark, C.E., and Clark, R.E. 2004. The medial temporal lobe. Annu. Rev. Neurosci. 27: 279-306.

Squire, L.R., Wixted, J.T., and Clark, R.E. 2007. Recognition memory and the medial temporal lobe: A new perspective. Nat. Review Neurosci. 8: 872-883.

Stark, C.E. and Squire, L.R. 2000. Recognition memory and familiarity judgments in severe amnesia: No evidence for a contribution of repetition priming. Behav. Neurosci. 114: 459-467.

Tulving, E. 1981. Similarity relations in recognition. J. Verb. Learn. Verb. Behav. 20: 479-496.

Tunney, R.J. and Fernie, G. 2007. Repetition priming affects guessing not familiarity. Behav. Brain Funct. 3: 40-46.

Verfaellie, M. and Cermak, L.S. 1999. Perceptual fluency as a cue for recognition judgments in amnesia. Neuropsychology 13: 198-205.

Wais, P.E., Wixted, J.T., Hopkins, R.O., and Squire, L.R. 2006. The hippocampus supports both the recollection and the familiarity components of recognition memory. Neuron 49: 459-466.

Weiskrantz, L., Barbur, J.L., and Sahraie, A. 1995. Parameters affecting conscious versus unconscious visual discrimination with damage to the visual cortex (V1). Proc. Natl. Acad. Sci. 92: 6122-6126.

Westerberg, C.E., Paller, K.A., Weintraub, S., Mesulam, M.M., Holdstock, J.S., Mayes, A.R., and Reber, P.J. 2006. When memory does not fail: Familiarity-based recognition in mild cognitive impairment and Alzheimer's disease. Neuropsychology 20: 193-205.

Whittlesea, B.W. and Price, J.R. 2001. Implicit/explicit memory versus analytic/nonanalytic processing: Rethinking the mere exposure effect. Mem. Cognit. 29: 234-246.

Whittlesea, B.W. and Williams, L.D. 2000. The source of feelings of familiarity: The discrepancy-attribution hypothesis. J. Exp. Psychol. Learn. Mem. Cogn. 26: 547-565.

Wiggs, C.L. and Martin, A. 1998. Properties and mechanisms of perceptual priming. Curr. Opin. Neurobiol. 8: 227-233.

Wixted, J.T. and Squire, L.R. 2004. Recall and recognition are equally impaired in patients with selective hippocampal damage. Cogn. Affect. Behav. Neurosci. 4: 58-66.

Yonelinas, A.P. 2001. Consciousness, control, and confidence: The 3 Cs of recognition memory. J. Exp. Psychol. Gen. 130: 361-379.

Yonelinas, A.P. 2002. The nature of recollection and familiarity: A review of 30 years of research. J. Mem. Lang. 46: 441-517.

Received February 19, 2008; accepted in revised form April 17, 2008. 


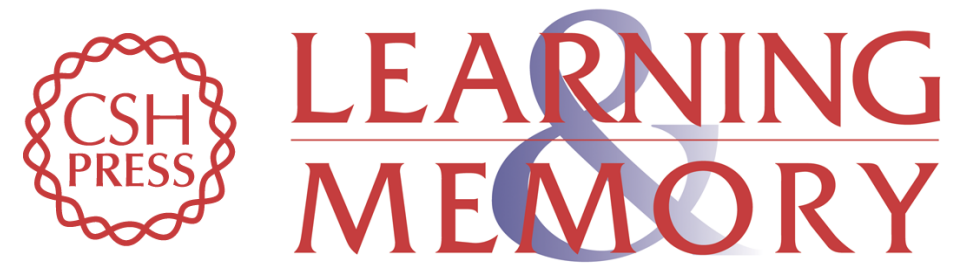

\section{Accurate forced-choice recognition without awareness of memory retrieval}

Joel L. Voss, Carol L. Baym and Ken A. Paller

Learn. Mem. 2008, 15:

Access the most recent version at doi:10.1101//m.971208

References This article cites 43 articles, 2 of which can be accessed free at:

http://learnmem.cshlp.org/content/15/6/454.full.html\#ref-list-1

License

Email Alerting Receive free email alerts when new articles cite this article - sign up in the box at the Service top right corner of the article or click here. 